\title{
Efficient evaluation of accuracy of molecular quantum dynamics using dephasing representation
}

\author{
Baiqing Li, ${ }^{a)}$ Cesare Mollica, and Jiří Vaníček ${ }^{\text {b) }}$ \\ Laboratory of Theoretical Physical Chemistry, Institut des Sciences et Ingénierie Chimiques, \\ École Polytechnique Fédérale de Lausanne (EPFL), CH-1015 Lausanne, Switzerland
}

(Received 15 May 2009; accepted 7 July 2009; published online 23 July 2009)

\begin{abstract}
$A b$ initio methods for the electronic structure of molecules have reached a satisfactory accuracy for calculations of static properties but remain too expensive for quantum dynamics calculations. We propose an efficient semiclassical method for evaluating the accuracy of a lower level quantum dynamics, as compared to a higher level quantum dynamics, without having to perform any quantum dynamics. The method is based on the dephasing representation of quantum fidelity and its feasibility is demonstrated on the photodissociation dynamics of $\mathrm{CO}_{2}$. Our accuracy test can be easily implemented in existing molecular dynamics codes, thus offering wide applicability. (C) 2009 American Institute of Physics. [DOI: 10.1063/1.3187240]
\end{abstract}

\section{INTRODUCTION}

$A b$ initio methods for the electronic structure of molecules have reached a satisfactory accuracy for calculations of static properties, such as energy barriers or force constants at local minima of the potential energy surface (PES). However, the most accurate of such methods remain out of reach when one wants to describe molecular properties that depend on the full quantum dynamics. ${ }^{1}$ To make a calculation feasible, one has to approximate the PES (Ref. 1) and/or the dynamics of the system, ${ }^{2,3}$ but both approaches can have nontrivial effects on the result. ${ }^{4}$ We consider only the first approach, in which quantum dynamics is done exactly but on a PES obtained by a lower level electronic structure method that is less accurate but also less expensive. When such a calculation is finished, its accuracy is not known because of the forbidding expense of the dynamics on the more accurate potential. In this communication, we propose an efficient and accurate semiclassical (SC) method for evaluating the accuracy of the lower level quantum dynamics without having to perform the higher level quantum dynamics. Our accuracy test requires the forces (at the lower level) and the potential energy values (at both levels), but only along certain classical (CL) trajectories. In particular, since our method does not even require computing the lower level quantum dynamics, it can also be used to justify, in advance, the investment of computational resources into the lower level calculations.

\section{METHODOLOGY}

We consider two PESs: (i) $V_{\text {acc }}$, a very accurate higherlevel electronic structure PES (presumably "almost exact"), which is too expensive to be used for quantum dynamics, and (ii) $V_{\text {appr }}$, an approximate PES, obtained by a lower-level electronic structure method and "cheap" enough to be used for quantum dynamics. Various quantities can describe dif-

\footnotetext{
${ }^{a}$ Present address: School of Chemistry and Chemical Engineering, Shandong University, Jinan, Shandong, 250100, P. R. China.

${ }^{b)}$ Electronic mail: jiri.vanicek@epfl.ch.
}

ferent time-dependent features of a quantum system, but a single quantity that includes all information about the system is the time-dependent wave function, $\psi(t)$. The accuracy of quantum dynamics on $V_{\text {appr }}$ can therefore be evaluated by computing the quantum-mechanical $(\mathrm{QM})$ overlap,

$$
f_{\mathrm{QM}}:=\left\langle\psi_{\mathrm{acc}}(t) \mid \psi_{\mathrm{appr}}(t)\right\rangle,
$$

where the subscript of $\psi$ indicates the PES used for propagating the initial state $\psi(0)$. The quantity $F(t):=\left|f_{\mathrm{QM}}(t)\right|^{2}$, known as quantum fidelity or Loschmidt echo, has been defined by Peres ${ }^{5}$ to measure the sensitivity of quantum dynamics to perturbations. Much effort has been devoted to the study of the temporal decay of fidelity and many universal regimes have been found. ${ }^{6}$ In our setting, if $F(t) \approx 1$ for all times up to $t_{\max }$, we can trust quantum dynamics on the approximate potential $V_{\text {appr }}$ and use the resulting $\psi_{\text {appr }}(t)$ to compute all dynamical properties up to $t_{\max }$. Since utilizing $V_{\text {acc }}$ is too expensive, $\psi_{\text {acc }}(t)$ cannot be computed. We describe a method which gives an accurate estimate of $f_{\mathrm{QM}}$ without having to compute $\psi_{\text {acc }}(t)$ nor $\psi_{\text {appr }}(t)$.

The method is based on the dephasing representation (DR) of quantum fidelity, a SC approximation proposed by one of us to evaluate fidelity in chaotic, integrable, and mixed systems even in nonuniversal regimes, which are sensitive to the initial state and details of dynamics. ${ }^{7,8}$ Presently, we are interested in a specific type of "perturbation," namely, the difference, $\Delta V=V_{\text {appr }}-V_{\text {acc }}$, between the approximate and accurate PESs. The DR of fidelity amplitude is an interference integral

$$
\begin{aligned}
& f_{\mathrm{DR}}(t):=\int d x^{0} \rho_{W}\left(x^{0}\right) \exp \left[-i \Delta S\left(x^{0}, t\right) / \hbar\right], \\
& \Delta S\left(x^{0}, t\right)=\int_{0}^{t} d \tau \Delta V\left[q_{\mathrm{acc}}^{\tau}\left(x^{0}\right)\right] .
\end{aligned}
$$

Here $x$ denotes a point $(q, p)$ in phase space, the superscript is the corresponding time, $\Delta S\left(x^{0}, t\right)$ is the action due to $\Delta V$ 
along the trajectory $q_{\text {acc }}^{t}\left(x^{0}\right)$ of $V_{\text {acc }}$, and $\rho_{W}$ is the Wigner function of the initial state $\psi$,

$$
\rho_{W}(x)=h^{-d} \int d \xi \psi^{*}\left(q+\frac{\xi}{2}\right) \psi\left(q-\frac{\xi}{2}\right) \exp \left(i \frac{\xi p}{\hbar}\right)
$$

In the dephasing representation, all of fidelity decay appears to be due to interference and none due to decay of CL overlaps.

Surprising accuracy of the DR was justified by the shadowing theorem, ${ }^{7}$ or, in the case of the initial state $\psi(0)$ supported by a Lagrangian manifold, by the structural stability of manifolds. ${ }^{9}$ Interestingly, validity of the DR goes much beyond validity of the SC approximation for quantum dynamics on $V_{\text {acc }}$ or $V_{\text {appr }}$. Qualitatively, this is due to the alleviation of the "sign problem" of quantum or SC dynamics: large actions needed for dynamics on $V_{\text {acc }}$ or $V_{\text {appr }}$ are replaced by much smaller actions $\Delta S$ needed for fidelity calculation. Rapid oscillations in the SC expression for the dynamics on $V_{\text {acc }}$ or $V_{\text {appr }}$ are replaced by much slower oscillations in the DR. In a chaotic system where $\sim 10^{35}$ trajectories would be needed for computing $\psi_{\text {acc }}(t)$ semiclassically, as few as 1000 trajectories were sufficient to compute fidelity amplitude. ${ }^{10}$ The accuracy of the DR was explored numerically in Refs. 7, 8, and 11. Errors in the DR can be estimated analytically, suggesting that the DR breaks down for very large perturbations, when the effective perturbation is larger than the square root of the effective Planck's constant. ${ }^{12}$ However, the DR remains accurate for fairly large perturbations, even when corresponding CL trajectories of $V_{\text {acc }}$ and $V_{\text {appr }}$ are completely different. $7,8,11$

The fundamental reason why quantum dynamics calculations are expensive is the nonlocality of quantum mechanics: The wave function $\psi(t+\Delta t)$ at any point in space depends in general on $\psi(t)$ in the whole space. There are many computational methods for quantum dynamics and they differ somewhat in their scaling. For demonstration purposes, we consider two methods that represent two very different general approaches.

The first approach starts with the construction of a global PES, with a computational cost $c n^{d}$, where $c$ is the cost of a single potential evaluation, $d$ is the number of degrees of freedom (DOF), and $n$ is the number of grid points in each DOF. Once the PES is known, dynamics can be performed, e.g., by the split-operator method. ${ }^{13}$ In this method, the quantum evolution operator for time step $\Delta t$ is approximated by

$$
e^{-i H \Delta t / \hbar}=e^{-i T \Delta t / \hbar} e^{-i V \Delta t / \hbar}+O\left(\Delta t^{2}\right),
$$

where $H=T+V$ is the Hamiltonian and $T$ is the kinetic energy operator. Quantum dynamics consists of alternate kinetic and potential propagations (which are just multiplications in momentum and coordinate representations, respectively) and a fast Fourier transform (FFT) to switch between these representations. The complexity of FFT is $O(N \log N)$, where $N=n^{d}$ gives the dimension of the Hilbert space, so the cost of propagation is $O\left(d t n^{d} \log n\right)$. Note that the same number of potential energy evaluations is required no matter how long the propagation is. Still, memory requirements make the storage of a global PES feasible only for small systems.
In the second approach, potential energy is calculated "on the fly" only in the vicinity of the propagated wave packet. At each time step, the cost is $c \widetilde{n}^{d} / 2 t_{1 / 2}$, where $\tilde{n}$ is the number of grid points in each DOF on which $\psi(t)$ is not negligible, and $t_{1 / 2}$ the average number of time steps after which the wavepacket moves sufficiently so that only half of the previously computed values of $V$ can be reused. (For simplicity, we neglect the small speedup gained by not having to recalculate the potential values during wavepacket recurrences.) Presumably, $\tilde{n} \ll n$, but the exponential scaling with $d$ remains. Assuming that $\log \tilde{n}<c$, the cost of actual propagation (e.g., by FFT) is negligible to the cost of potential evaluation and the overall cost of the dynamics is $O\left(c \tilde{n}^{d} t / 2 t_{1 / 2}\right)$.

In the DR, potential energy and forces are also evaluated on the fly, but along CL trajectories. At each time step, the cost is only $O\left(d c n_{\text {paths }}\right)$, where $n_{\text {paths }}$ is the number of CL trajectories used. The "hidden cost" in usual SC approximations is the strong dependence of $n_{\text {paths }}$ on $t, d$, or the type of dynamics. In Ref. 8, it was shown rigorously that $n_{\text {paths }}=C\left(F_{\mathrm{DR}}\right) \sigma^{-2}$, where $F_{\mathrm{DR}}$ is the value of fidelity one wants to simulate, $\sigma$ is the error (due to finite $n_{\text {paths }}$ ) that one wants to reach, and $0 \leq C \leq 3$. Consequently, for given $F_{\mathrm{DR}}$ and $\sigma$, the required number of trajectories is independent of $t, d$, or the type of dynamics! It was also shown in Ref. 8 that when $F \rightarrow 1$, which is the most interesting in our application, $C \rightarrow 0$, i.e., $n_{\text {paths }}$ needed for convergence of $F_{\mathrm{DR}}$ becomes even smaller. The overall cost of the DR dynamics is $O\left(d c C \sigma^{-2} t\right)$. In particular, there is no exponential scaling with the number of DOF or time.

Clearly, the DR is faster than the construction of a global PES and than the quantum dynamics on the fly. Only at very long times $t$, in the first quantum approach, since the global PES is already constructed, does the cost of propagation become dominant and the QM method competes with the DR one, which requires new potential evaluations. However even then the ratio of the costs of DR and QM is $c n_{\text {paths }} / n^{d} \log n$. If the number of nuclear DOF is comparable to the number of electronic DOF, then even for the most accurate $a b$ initio methods (e.g., the coupled clusters), $c$ scales polynomially with $d$, and so for large enough $d$, DR will remain faster than the QM dynamics.

The DR calculation can be further accelerated by exchanging the roles of $V_{\text {acc }}$ and $V_{\text {appr }}$. We will denote by DR 1 the DR expression defined in Eqs. (2) and (3), and by DR2 an analogous expression,

$$
\begin{aligned}
& f_{\mathrm{DR} 2}(t):=\int d x^{0} \rho_{W}\left(x^{0}\right) \exp \left[i \Delta S\left(x^{0}, t\right) / \hbar\right] \\
& \Delta S\left(x^{0}, t\right)=\int_{0}^{t} d \tau \Delta V\left[q_{\text {appr }}^{\tau}\left(x^{0}\right)\right] .
\end{aligned}
$$

DR2 denotes the DR computed as an interference integral due to the action of $-\Delta V$ along the trajectory of $V_{\text {appr }}$. From definition (1), it is clear that exchanging $V_{\text {acc }}$ and $V_{\text {appr }}$ results in complex conjugation of $f_{\mathrm{QM}}$ and has no effect on $F_{\mathrm{QM}}$. In 
principle, there could be an effect on $f_{\mathrm{DR}}$ because expression (2) does not have such symmetry, but numerical evidence presented below shows that $\mathrm{DR} 1 \approx \mathrm{DR} 2$, providing further support for the approximation. If efficiency is important, one should choose DR2 over DR1 since DR2 requires values and gradients of the "cheaper" PES ( $\left.V_{\text {appr }}\right)$, but only values of the more expensive PES $\left(V_{\text {acc }}\right)$, whereas DR1 requires values of $V_{\text {appr }}$, but both values and gradients of $V_{\text {acc }}$. If calculation of DR1 is affordable, comparison of DR1 and DR2 results can be used as an applicability test of the DR method since comparison with $f_{\mathrm{QM}}$ will not be available (computation of $f_{\mathrm{QM}}$ would require full quantum dynamics on $\left.V_{\text {acc }}\right)$. A large difference between the DR1 and DR2 results would be a sign of the breakdown of DR. So the requirement $F_{\mathrm{DR} 1} \approx F_{\mathrm{DR} 2}$ is a necessary but not a sufficient condition for the applicability of the DR.

One could object that since the DR is an intrinsically SC approximation, it might suffice to estimate fidelity classically. We explored this idea by comparing quantum fidelity with its CL analog, called CL fidelity, ${ }^{14}$ defined in our case by

$$
F_{\mathrm{CL}}(t):=h^{d} \int d x \rho_{\mathrm{CL}, \mathrm{acc}}^{t}(x) \rho_{\mathrm{CL}, \mathrm{appr}}^{t}(x)
$$

where $\rho_{\mathrm{CL}}^{t}$ is the CL phase-space density evolved with the indicated potential to time $t$. For initial Gaussian wave packets, $\rho_{\mathrm{CL}}^{0}=\rho_{W}^{0}$. Unlike the DR, CL fidelity does not include any dynamical quantum effects and below we show that indeed $F_{\text {CL }}$ gives much worse results.
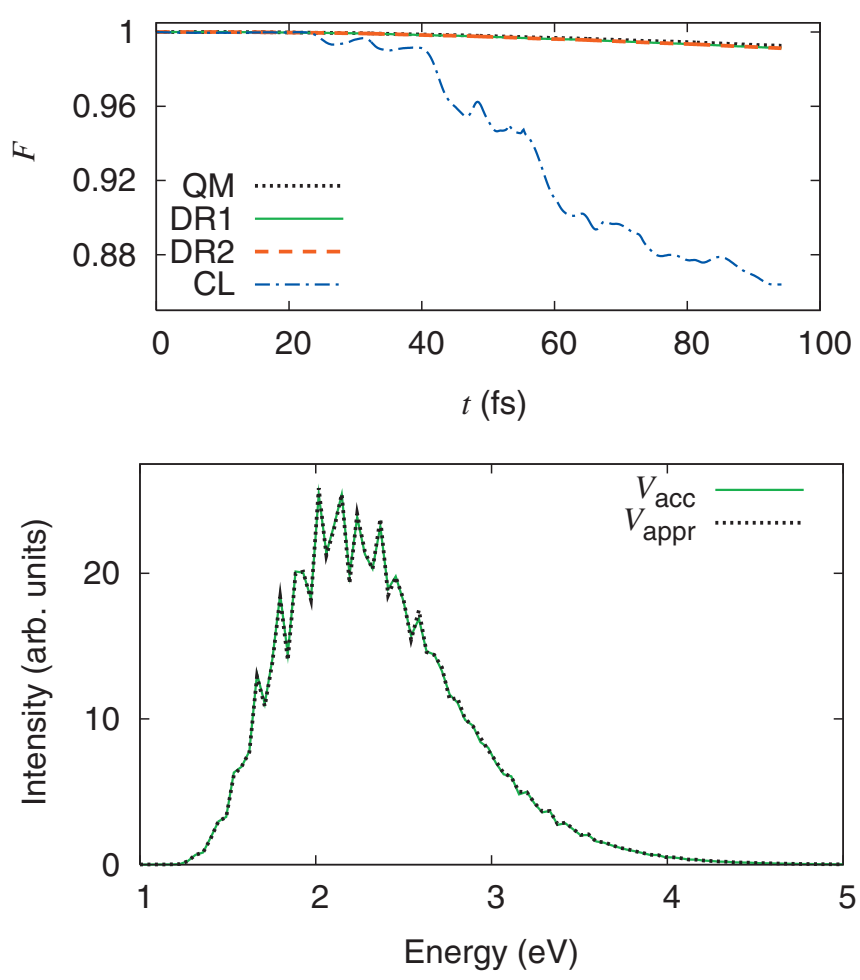

FIG. 1. Fidelity decay (top) and spectrum change (bottom) for $D_{e, \text { CO }}^{\prime}$ $=D_{e, \mathrm{CO}}+0.02 \mathrm{eV}=11.26 \mathrm{eV}$.

\section{RESULTS}

To show the feasibility of our method, we applied it to the photodissociation dynamics of a collinear carbon dioxide molecule, a model that had been studied extensively by both quantum-dynamical $^{15}$ and SC methods. ${ }^{16}$ Invoking the Franck-Condon principle, the photodissociation process is described by the quantum dynamics of the initial state (vibrational ground state of the electronic ground state PES) on the dissociative excited PES. One can obtain the photodissociation spectrum simply by taking the Fourier transform of the autocorrelation function $C(t):=\langle\psi(0) \mid \psi(t)\rangle$. In future applications, one will not be able to find $\psi_{\text {acc }}(t)$ and $f_{\mathrm{QM}}(t)$ due to the prohibitive computational expense. Here, in order to demonstrate the accuracy of the method, we want to compare $f_{\mathrm{DR}}(t)$ with $f_{\mathrm{QM}}(t)$ and so we define $V_{\mathrm{acc}}:=V_{\mathrm{LEPS}}$ to be the analytical LEPS potential for $\mathrm{CO}_{2}{ }^{15,17}$ and $V_{\text {appr }}:=V_{\text {LEPS }}^{\prime}$ to be the LEPS potential with one of the parameters perturbed. Specifically, we change either the equilibrium bond length $R_{e, \mathrm{CO}}$ or the bond dissociation energy $D_{e, \mathrm{CO}}$ of the CO bond. We imagine that we would like to obtain a spectrum corresponding to $V_{\text {acc }}$ but can only afford quantum dynamics on $V_{\text {appr }}$. By estimating quantum fidelity amplitude $f_{\mathrm{QM}}$ by $f_{\mathrm{DR}}$, we can determine whether we can trust the spectrum computed using quantum dynamics on $V_{\text {appr. }}$. From another perspective, we can use $f_{\mathrm{DR}}$ to evaluate how errors in experimental values of $R_{e, \mathrm{CO}}$ and $D_{e, \mathrm{CO}}$ affect the computed spectrum.

Figure 1 shows an example where $V_{\text {LEPS }}^{\prime}$ has a perturbed bond dissociation energy, $D_{e, \mathrm{CO}}^{\prime}=D_{e, \mathrm{CO}}+0.02 \mathrm{eV}$ $=11.26 \mathrm{eV}$. The figure shows that three approaches to compute fidelity (QM, DR1, and DR2) give very similar results.
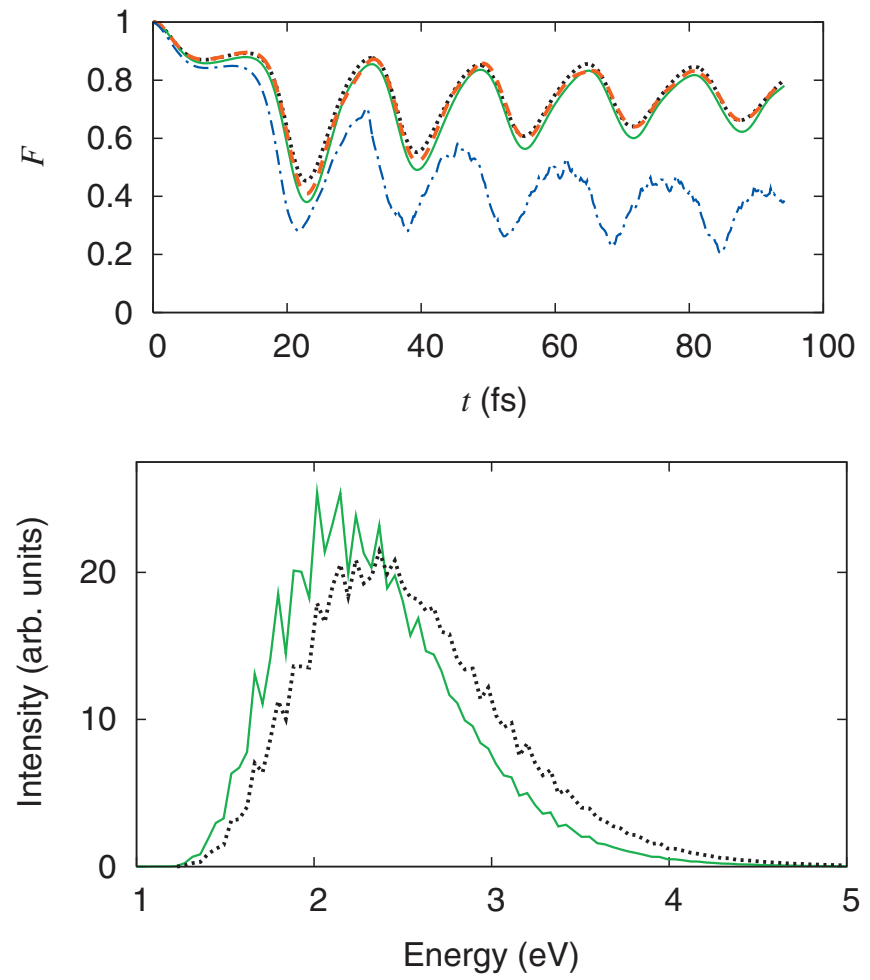

FIG. 2. Fidelity decay (top) and spectrum change (bottom) for $R_{e, \mathrm{CO}}^{\prime}$ $=R_{e, \mathrm{CO}}+0.02 a_{0}=2.15 a_{0}$. The notation is as in Fig. 1 . 
This turns out to be a small perturbation since fidelity remains close to unity, $F_{\mathrm{QM}} \geq 0.99$. Therefore we should be able to trust the spectrum computed using $\psi_{\text {appr }}(t)$. This is justified in the bottom panel where spectra corresponding to $V_{\text {acc }}$ and $V_{\text {appr }}$ prove to be almost identical. (The spectrum is significantly affected for $\Delta D_{e, \mathrm{CO}} \gtrsim 0.1 \mathrm{eV}$.) Unlike the DR, CL fidelity decays much faster than its quantum analog. Judging by $F_{\mathrm{CL}}$ only, one would conclude incorrectly that the spectrum computed using $V_{\text {appr }}$ should not be trusted. Partially constructive interference, captured by DR, prevents quantum fidelity from decaying with the fast rate of CL fidelity decay.

Figure 2 shows an example where $V_{\text {LEPS }}^{\prime}$ has a perturbed equilibrium bond length, $R_{e, \mathrm{CO}}^{\prime}=R_{e, \mathrm{CO}}+0.02 a_{0}=2.15 a_{0}$. The figure shows that $F_{\mathrm{DR}}$ agrees with $F_{\mathrm{QM}}$ and even reproduces detailed oscillations of quantum fidelity. This turns out to be a large perturbation since fidelity falls below the value of 0.5 . We therefore expect the spectrum computed using $\psi_{\text {appr }}(t)$ to differ significantly from the "true" spectrum computed using $\psi_{\text {acc }}(t)$. Indeed, the bottom panel shows that the spectrum of $V_{\text {appr }}$ is shifted and has different peak intensities than the spectrum of $V_{\text {acc }}$. Figure 2 also shows that for large $\Delta V$, CL fidelity starts to behave similarly to quantum fidelity, but is still much worse than DR.

All calculations were performed for 600 time steps of $0.16 \mathrm{fs}$ each. Converged quantum calculations required $n=1024$ points to discretize each $\mathrm{CO}$ bond length from 0 to $20 a_{0} \approx 10.6 \AA$, altogether using a $1024 \times 1024$ grid to represent the PES and the wave function. The DR calculation converged fully with $n_{\text {paths }}=512$ trajectories (shown in Figs. 1 and 2), but a much smaller value, $n_{\text {paths }}=64$, already gives very accurate results sufficient for our application (not shown). Since our approach requires trajectories of $V_{\text {appr }}$ and potential energy values of $V_{\text {appr }}$ and $V_{\text {acc }}$ along these trajectories, the overall computational cost is between the costs of molecular dynamics on $V_{\text {appr }}$ and $V_{\text {acc. }}$. In any case, the cost will be much lower than the cost of the quantum dynamics on $V_{\text {acc }}$. In Figs. 1 and 2, the costs of QM, DR, and CL calculations were, respectively, 3187, 5, and $862 \mathrm{~s}$. (The reason why $F_{\mathrm{CL}}$ costs more than $F_{\mathrm{DR}}$ is that the calculation of $F_{\mathrm{CL}}$ scales with $t^{2}$ : the explanation is beyond the scope of this paper.) Both figures show fidelity $F$, rather than fidelity amplitude $f$, which is a complex number containing more information. If $F \ll 1$, clearly our approximate dynamics is not sufficient. However, if $F \approx 1$, the spectrum could still be affected by a time-dependent phase of $f$. In such cases one should also examine fidelity amplitude.

\section{CONCLUSION}

Our fidelity calculation for the photodissociation of $\mathrm{CO}_{2}$ is to our knowledge the first fidelity calculation for a realistic chemical system and it is reassuring that the DR remains valid. ${ }^{6}$ Clearly, in chemical physics, one is interested in systems with many more than two DOF. However, already in the simple $\mathrm{CO}_{2}$ system, the DR calculation of fidelity was more than 100 times faster than the exact quantum calculation. We expect that in larger systems, much larger speedups could be achieved.

The information required in a DR calculation is similar to the information needed in molecular dynamics (MD) calculations. Implementation of DR into any MD code would require a single addition: calculation of the action $\Delta S$. However there is an important difference between the DR and MD: in the DR, nuclear quantum effects are included at least approximately, whereas in MD, even if an ab initio electronic potential is used, they are completely lost. This can be clearly seen in Figs. 1 and 2 where $F_{\mathrm{DR}} \approx F_{\mathrm{QM}} \neq F_{\mathrm{CL}}$ since $F_{\mathrm{CL}}$ is basically a MD calculation of fidelity. With little effort, MD codes that currently compute only CL nuclear dynamics, could be used for evaluating the accuracy of quantum dynamics on the same PES.

To conclude, we found a promising method to estimate the accuracy of quantum dynamics on an approximate potential. However, the DR approach is applicable to other types of perturbations than discrepancies in the PES. For example, the DR could be used to evaluate how laser pulse noise affects quantum control ${ }^{18}$ or how perturbations affect quantum computation.

\section{ACKNOWLEDGMENTS}

This research was supported by the Swiss NSF (Grant No. 200021_124936/1) and by the EPFL. We thank Tomáš Zimmermann for useful discussions.

${ }^{1}$ D. C. Clary, Science 321, 789 (2008).

${ }^{2}$ N. Makri, Annu. Rev. Phys. Chem. 50, 167 (1999).

${ }^{3}$ W. H. Miller, Proc. Natl. Acad. Sci. U.S.A. 102, 6660 (2005).

${ }^{4}$ H. Rabitz, Chem. Rev. (Washington, D.C.) 87, 101 (1987).

${ }^{5}$ A. Peres, Phys. Rev. A 30, 1610 (1984).

${ }^{6}$ T. Gorin, T. Prosen, T. H. Seligman, and M. Žnidarič, Phys. Rep. 435, 33 (2006).

${ }^{7}$ J. Vaníček, Phys. Rev. E 70, 055201(R) (2004).

${ }^{8}$ J. Vaníček, Phys. Rev. E 73, 046204 (2006).

${ }^{9}$ N. R. Cerruti and S. Tomsovic, Phys. Rev. Lett. 88, 054103 (2002).

${ }^{10}$ J. Vaníček and E. J. Heller, Phys. Rev. E 68, 056208 (2003).

${ }^{11}$ J. Vaníček, e-print arXiv:quant-ph/0410205.

${ }^{12}$ J. Vaníček, C. Mollica, T. Prosen, and W. Strunz (unpublished).

${ }^{13}$ M. D. Feit, J. J. A. Fleck, and A. Steiger, J. Chem. Phys. 47, 412 (1982).

${ }^{14}$ T. Prosen and M. Žnidarič, J. Phys. A 35, 1455 (2002).

${ }^{15}$ K. C. Kulander and J. C. Light, J. Chem. Phys. 73, 4337 (1980).

${ }^{16}$ T. Van Voorhis and E. J. Heller, Phys. Rev. A 66, 050501(R) (2002).

${ }^{17}$ S. Sato, J. Chem. Phys. 23, 592 (1955).

${ }^{18}$ B. Li, G. Turinici, V. Ramakrishna, and H. Rabitz, J. Phys. Chem. B 106, 8125 (2002) 